\title{
Seeking Supersolidity in Helium Layers
}

\section{A scheme that proves the superfluidity of a layer of helium-4 on graphite holds promise for demonstrating that the layer may also be a supersolid.}

\author{
By Rahul Rao
}

$\square$

hysicists believe that thin films of helium-4 painted upon a canvas of graphite may host a wellspring of exotic quantum effects, including supersolidity-a phenomenon in which a material behaves both as a solid with ordered structure and as a superfluid flowing with zero viscosity. Now, Jaewon Choi and colleagues at the Korea Advanced Institute of Science and Technology (KAIST), South Korea, have reported a promising step in the search for supersolidity in this system. They developed a setup that proves the helium-4-layer superfluidity while removing signals from unrelated effects that might have plagued previous experiments [1].

Physicists have been tinkering with helium-4-on-graphite for decades. Typically, they stacked several layers of helium-4 atop graphite disks placed on a torsional oscillator. As they twisted the disks, they saw shifts in the disks' oscillation periods that indicated that some of the layers were superfluid. Much effort has focused on studying the second in the helium-layer series because theory suggests that, in this layer, superfluidity could

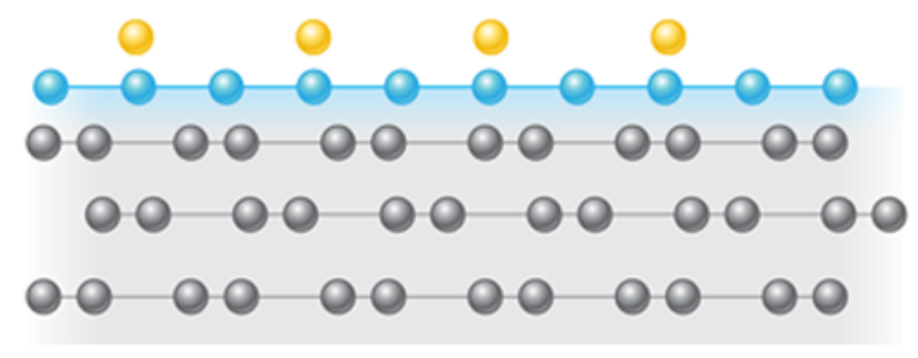

coexist with solidity. Proving supersolidity in experiments, however, is complicated by the fact that the signals are affected by factors unrelated to the quantum phase, such as imperfections in the torsional oscillator or anomalies in the elasticity of helium.

To overcome this problem, the KAIST researchers designed a novel torsional oscillator that, by operating at two frequencies, filters out such unwanted contributions to the signals. The team says that the experiments provided the clearest indication to date that the second layer is superfluid. They also say that the phase diagram derived for the second layer offers tantalizing hints that this layer could be a supersolid, but further research is needed to assess this hypothesis.

Rahul Rao is a freelance science writer based in New York.

\section{REFERENCES}

1. J. Choi et al., "Spatially modulated superfluid state in two-dimensional ${ }^{4}$ He films," Phys. Rev. Lett. 127, 135301 (2021).

Credit: APS/Alan Stonebraker 